\title{
STRICTLY CYCLIC OPERATOR ALGEBRAS
}

\author{
JOHN FROELICH
}

\begin{abstract}
We prove several results about the lattice of invariant subspaces of general strictly cyclic and strongly strictly cyclic operator algebras. A reflexive operator algebra $A$ with a commutative subspace lattice is strictly cyclic iff $\operatorname{Lat}(A)^{\perp}$ contains a finite number of atoms and each nonzero element of $\operatorname{Lat}(A)^{\perp}$ contains an atom. This leads to a characterization of the $n$-strictly cyclic reflexive algebras with a commutative subspace lattice as well as an extensive generalization of D. A. Herrero's result that there are no triangular strictly cyclic operators. A reflexive operator algebra $A$ with a commutative subspace lattice is strongly strictly cyclic iff $\operatorname{Lat}(A)$ satisfies A.C.C.

The distributive lattices which are attainable by strongly strictly cyclic reflexive algebras are the complete sublattices of $\{0,1] \times\{0,1\} \times \cdots$ which satisfy A.C.C.

We also show that if $\operatorname{Alg}(\mathscr{L})$ is strictly cyclic and $\mathscr{L} \subseteq$ atomic m.a.s.a. then $\operatorname{Alg}(\mathscr{L})$ contains a strictly cyclic operator.
\end{abstract}

\section{INTRODUCTION}

In this paper we study Hilbert space operator algebras $A$ which admit a strictly cyclic vector in the sense that the linear manifold $A x=\{T x: T \in$ $A\}=H$. For algebras acting on finite-dimensional spaces, every cyclic vector is strictly cyclic, but this is not the case in infinite dimensions. Previous studies [2, $7,8,10,13$ ] have tended to emphasize the topological, algebraic, and reflexivity consequences of a strictly cyclic vector, whereas we shall be concerned with the implications that a strictly cyclic vector has for the "global" structure of the algebra and its lattice of invariant subspaces.

In the first section we prove several results about the lattice of invariant subspaces of a general strictly cyclic operator algebra. This allows us to completely characterize the $n$-strictly cyclic reflexive operator algebras with a commutative subspace lattice. We indicate how one may deduce an extensive generalization of D. A. Herrero's result [9] that there are no triangular strictly cyclic operators as a corollary.

After characterizing a class of strongly strictly cyclic operator algebras in $\S 2$, we obtain a "model" for the commutative subspace lattices which are attainable by a strongly strictly cyclic reflexive algebra. This result is motivated by W. Arveson's characterization [1] of the abstract distributive lattices which are

Received by the editors December 22, 1988 and, in revised form, April 3, 1989.

1980 Mathematics Subject Classification (1985 Revision). Primary 47O25; Secondary 47A15. 
attainable by reflexive operator algebras, although our approach is quite different.

Lastly, we take up the question of when a strictly cyclic operator algebra actually contains a strictly cyclic operator. We close with some direction for further study.

A uniformly closed algebra $A$ is called $n$-strictly cyclic if there are vectors $x_{1}, \ldots, x_{n} \in H$ such that $H=A x_{1}+A x_{2}+\cdots+A x_{n}$, this being a sum of linear manifolds. An operator is called $n$-strictly cyclic if the uniformly (or weakly) closed algebra generated by $T$ is $n$-strictly cyclic, and it is called rationally $n$-strictly cyclic if the algebra generated by the rational functions in $T$, with poles off $\sigma(T)$, is $n$-strictly cyclic. A subspace lattice is a lattice of projections (containing 0,1 ) that is closed in the strong operator topology. We will let $\bigwedge P_{i}$ and $\bigvee P_{i}$ denote the infimum and supremum (respectively) of a family $\left\{P_{i}\right\}$ of projections. We shall frequently ideritify a closed subspace of $H$ with its orthogonal projection. If $A$ is an algebra of operators, then $\operatorname{Lat}(A)$ is the lattice of invariant subspaces, and if $\mathscr{L}$ is a subspace lattice, $\operatorname{Alg}(\mathscr{L})$ is the algebra of operators which leave each member of $\mathscr{L}$ invariant. An operator algebra is called reflexive if $\operatorname{Alg} \operatorname{Lat}(A)=A$. For a detailed study of these algebras, see [1, 4, and 14]. All Hilbert spaces are separable.

\section{Strictly CyClic Operator algebras}

Theorem 1.1. Let $A$ be an operator algebra with strictly cyclic vector $e$, and let $\mathscr{L} \subseteq \operatorname{Lat}(A)$ be a subspace lattice. Then there is a constant $\delta>0$ such that for any $F \in \mathscr{L}^{\perp}, F \neq 0, \delta \leq\|F e\|$.

Proof. Following Hopenwasser [11], we conclude that for any $x \in H$, the quantity $\operatorname{Sup}_{E \in \mathscr{L}}\left\|E^{\perp} x\right\| /\left\|E^{\perp} e\right\|<\infty$. This may be written as

$$
\operatorname{Sup}_{F \in \mathscr{L}^{\perp}, F \neq 0}\|F x\| /\|F e\|<\infty .
$$

For fixed $F \in \mathscr{L}^{\perp}, F \neq 0$, consider the operator

$$
x \rightarrow \frac{1}{\|F e\|} F x .
$$

By the uniform boundedness principle there is a constant $K$ such that

$$
\frac{1}{\|F e\|}\|F x\| \leq K\|x\|
$$

for any $F \in \mathscr{L}^{\perp}, F \neq 0$. Therefore $1=\|F\| \leq K\|F e\|$. It follows that $0<\delta=\inf _{F \in \mathscr{L}^{\perp}, F \neq 0}\|F e\|$.

Theorem 1.2. Let $\mathscr{L}$ be a subspace lattice contained in $\operatorname{Lat}(A)$, where $A$ is a strictly cyclic operator algebra. Then every nonzero element of $\mathscr{L}^{\perp}$ contains an atom of $\mathscr{L}^{\perp}$.

Proof. Let $F \in \mathscr{L}^{\perp}$ be nonzero. By Zorn's lemma there is a maximal chain $\mathscr{C}$ of nonzero projections $Q \in \mathscr{L}^{\perp}$ such that $Q \leq F$. Let $e$ be a strictly cyclic 
vector for $A$. If $\bigwedge \mathscr{C}=0$ then there is a sequence $Q_{1}>Q_{2}>\cdots$ in $\mathscr{C}$ with $\bigwedge_{n=1}^{\infty} Q_{n}=0$. Then $Q_{n} \stackrel{s}{\longrightarrow} 0$. This contradicts $\delta \leq\left\|Q_{n} e\right\|$ for some $\delta>0$. Hence $\Lambda \mathscr{C}$ is an atom of $\mathscr{L}^{\perp}$ contained in $F$.

The above is stronger than the known result [9] that every invariant subspace of $A$ is contained in a maximal invariant subspace, since we do not know a priori that a maximal $Q$ (coming from Zorn's lemma) containing $P \in \mathscr{L}$ is in $\mathscr{L}$.

Recall that a reflexive algebra with a commutative subspace lattice $\mathscr{L}$ has the form $\operatorname{Alg}(\mathscr{L})$.

Theorem 1.3. Let $\mathscr{L}$ be a commutative subspace lattice. Then $\operatorname{Alg}(\mathscr{L})$ is strictly cyclic iff $\mathscr{L}^{\perp}$ contains a finite number of atoms and every nonzero element of $\mathscr{L}^{\perp}$ contains an atom.

Proof. Assume that $A=\operatorname{Alg}(\mathscr{L})$ is strictly cyclic with strictly cyclic vector $e$. By Theorem 1.2, every nonzero element of $\mathscr{L}^{\perp}$ contains an atom. Let $F_{1}, F_{2}, \ldots$ be the atoms. For $i \neq j$ we have $F_{i} \perp F_{j}$. By Theorem 1.1 there is a constant $\delta>0$ such that $\delta \leq\left\|F_{i} e\right\|$. Bessel's inequality implies that

$$
n \delta^{2} \leq\left\|F_{1} e\right\|^{2}+\cdots+\left\|F_{n} e\right\|^{2} \leq\|e\|^{2} .
$$

Hence $n \leq\|e\|^{2} / \delta^{2}$. Thus the number of atoms is finite.

Conversely, let $F_{1}, F_{2}, \ldots, F_{n}$ be the atoms in $\mathscr{L}^{\perp}$. Choose unit vectors $e_{i}$ such that $F_{i} e_{i}=e_{i}$. Let $S$ be the projection onto the subspace $A \overline{e_{1}}+\cdots+$ $A \overline{e_{n}}$. The sum is closed since the projections onto the subspaces $A \overline{e_{i}}$ commute. Furthermore, since $S^{\perp} e_{i}=0, S^{\perp} \wedge F_{i}=0$ for each $i$. Hence $S^{\perp}=0$ so $S=I$. Therefore, every $x \in H$ may be written as $x=x_{1}+\cdots+x_{n}$ where $x_{i} \in A \overline{e_{i}}$. Now consider the operator $T=\sum_{i=1}^{n} x_{i} \otimes e_{i}$. Since $e_{i} \perp e_{j}$ for $i \neq j$, it is clear that $T e=x$, where $e=e_{1}+\cdots+e_{n}$. We need to show that $T \in \operatorname{Alg}(\mathscr{L})$. Let $P \in \mathscr{L}$. Then

$$
P^{\perp} T P=\sum_{i=1}^{n} P^{\perp} x_{i} \otimes P e_{i} .
$$

We will show that $P^{\perp} x_{i} \otimes P e_{i}=0$. Since $P^{\perp} \in \mathscr{L}^{\perp}$, either $F_{i}=F_{i} \wedge P^{\perp}$ or $0=F_{i} \wedge P^{\perp}$. In the first case, $P e_{i}=0$. In the second case, $F_{i} \leq P$ so $A \overline{e_{i}}$ is contained in the subspace corresponding to $P$. Hence $P^{\perp} x_{i}=0$. Thus $P^{\perp} T P=0$ for all $P \in \mathscr{L}$ and $T \in \operatorname{Alg}(\mathscr{L})$.

The converse could have been proved by applying [11] to $e_{1}+\cdots+e_{n}$. The above direct proof gives the following corollary.

Corollary 1.4. Let $\operatorname{Alg}(\mathscr{L})$ be strictly cyclic with strictly cyclic vector $e$. Then there is an $n$ such that for any $x \in H$, the equation $T e=x$ can be solved by an operator $T \in \operatorname{Alg}(\mathscr{L})$ with $\operatorname{rank}(T) \leq n$. 
Proof. We know there is a strictly cyclic vector $v$ for which the corollary is true. We have $T v=x$ with $\operatorname{rank}(T) \leq n$. For some $R \in \operatorname{Alg}(\mathscr{L})$ we have $R e=v$. So $T R e=x$ with $\operatorname{rank}(T R) \leq n$.

In general, $\operatorname{Alg}(\mathscr{L})$ need not contain any finite rank or even compact operators [5]. Theorem 1.3 gives a complete description of the strictly cyclic reflexive operator algebras with a commutative subspace lattice. We would now like to give several illustrative examples.

Example 1. Let $e_{1}, e_{2}, \ldots$ be an orthonormal basis for $H$. Let $P_{n}=$ $\overline{s p}\left\{e_{n}, e_{n+1}, \ldots\right\}$ and $P_{\infty}=0$. Take $N^{\perp}=\left\{P_{n}: n=0,1,2, \ldots\right\}$. Then $N^{\perp}$ is a commutative subspace lattice and $\operatorname{Alg}\left(N^{\perp}\right)$ is the (nest) algebra of lower triangular matrices.

$$
\left[\begin{array}{ccccc}
a_{11} & 0 & \ldots & & \\
a_{21} & a_{22} & 0 & \ldots & \\
a_{31} & a_{32} & a_{33} & 0 & \ldots \\
\cdot & \cdot & \cdot & & \\
\cdot & \cdot & \cdot & &
\end{array}\right]
$$

This operator algebra is strictly cyclic, whereas the algebra of upper triangular matrices $\operatorname{Alg}(N)$ is not. This is because $N^{\perp}$ contains no atoms.

Example 2. We consider a "continuous" version of the previous example. On $L^{2}[0,1]$ with Lebesgue measure, let $P_{t}$ be the projection onto the subspace of functions concentrated on the interval $[t, 1]$, and let $\mathscr{V}=\left\{P_{t}: 0 \leq t \leq 1\right\}$. The lattice $\mathscr{V}$ is again a nest, and $\operatorname{Alg}(\mathscr{V})$, the so-called Volterra nest algebra, consists of all operators whose support [1] lies in the triangle shown in Figure 1. Since $\mathscr{V}^{\perp}$ contains no atoms, $\operatorname{Alg}(\mathscr{V})$ is not strictly cyclic. In particular it contains no strictly cyclic operator.

We now turn to the problem of determining when $\operatorname{Alg}(\mathscr{L})$ is $n$-strictly cyclic for a commutative subspace lattice $\mathscr{L}$.

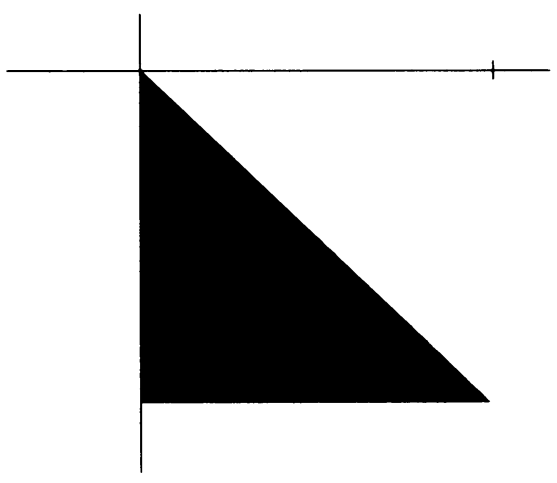

FIGURE 1 
Given a subspace lattice $\mathscr{L}$, we define the subspace lattice $\mathscr{L} \otimes I_{n}$ to be the lattice of $n \times n$ operator matrices

$$
\left\{\left[\begin{array}{lll}
P & \cdot & \\
& & 0 \\
0 & & P
\end{array}\right]: P \in \mathscr{L}\right\} .
$$

The following is well known: $M_{n}(A)=\operatorname{Alg}\left(\mathscr{L} \otimes I_{n}\right)$.

Proof. Clearly, $M_{n}(A) \subseteq \operatorname{Alg}\left(\mathscr{L} \otimes I_{n}\right)$. If $\left[A_{i j}\right] \in \operatorname{Alg}\left(\mathscr{L} \otimes I_{n}\right)$ then

$$
\left[\begin{array}{cccc}
P^{\perp} & & & \\
& P^{\perp} & & 0 \\
& & \cdot & \\
0 & & & P^{\perp}
\end{array}\right]\left[\begin{array}{ccc}
A_{11} & A_{12} & \cdots \\
A_{21} & A_{22} & \cdots \\
\cdot & \cdot & \\
\cdot & \cdot &
\end{array}\right]\left[\begin{array}{cccc}
P & & \\
& P & & 0 \\
& & \cdot & \\
0 & & P
\end{array}\right]=0 .
$$

So $P^{\perp} A_{i j} P=0$ for all $P \in \mathscr{L}$. Thus $A_{i j} \in \operatorname{Alg}(\mathscr{L})=A$ and so $\left[A_{i j}\right] \in$ $M_{n}(A)$.

Proposition 1.6. An operator algebra $A$ is $n$-strictly cyclic iff the operator algebra $M_{n}(A)$ is strictly cyclic.

Proof. Assume $M_{n}(A)$ is strictly cyclic. Let $y \in H$. Then there is a matrix $\left[A_{i j}\right]$ and vector

$$
\left[\begin{array}{c}
x_{1} \\
\cdot \\
x_{n}
\end{array}\right]
$$

such that

$$
\left[\begin{array}{ccc}
A_{11} & \cdots & A_{1 n} \\
\cdot & & \cdot \\
A_{n 1} & \cdots & A_{n n}
\end{array}\right]\left[\begin{array}{c}
x_{1} \\
\cdot \\
x_{n}
\end{array}\right]=\left[\begin{array}{l}
y \\
0 \\
0
\end{array}\right]
$$

So $A_{11} x_{1}+\cdots+A_{1 n} x_{n}=y$. Thus $A$ is $n$-strictly cyclic. The converse follows by solving each row of $\left[A_{i j}\right]$ for the vector

$$
\left[\begin{array}{c}
y_{1} \\
\cdot \\
y_{n}
\end{array}\right]
$$

Theorem 1.7. Let $\mathscr{L}$ be a commutative subspace lattice. If $\operatorname{Alg}(\mathscr{L})$ is $n$-strictly cyclic, then $\operatorname{Alg}(\mathscr{L})$ is strictly cyclic.

Proof. If $A=\operatorname{Alg}(\mathscr{L})$ is $n$-strictly cyclic, then $M_{n}(A)=\operatorname{Alg}\left(\mathscr{L} \otimes I_{n}\right)$ is strictly cyclic. Therefore $\left(\mathscr{L} \otimes I_{n}\right)^{\perp}=\mathscr{L}^{\perp} \otimes I_{n}$ has a finite number of atoms and every nonzero element of $\mathscr{L}^{\perp} \otimes I_{n}$ contains an atom. Since $\mathscr{L}^{\perp}$ is lattice isomorphic to $\mathscr{L}^{\perp} \otimes I_{n}$, the same is true for $\mathscr{L}^{\perp}$. Therefore $\operatorname{Alg}(\mathscr{L})$ is strictly cyclic.

As a corollary we may deduce generalizations of D. A. Herrero's result that there are no strictly cyclic upper triangular operators [9]. In what follows, $\operatorname{Alg}(Z)$ is the algebra of two-way upper triangular matrices. 


\section{Corollary 1.8.}

(i) $\operatorname{Alg}(N)$ contains no rationally $n$-strictly cyclic operators.

(ii) $\operatorname{Alg}(Z)$ contains no $n$-strictly cyclic operators.

(iii) $\operatorname{Alg}(\mathscr{V})$ contains no $n$-strictly cyclic operators.

Proof. The proof of (i) is immediate once we recall that $\operatorname{Alg}(N)$ in inverse closed.

It is known that $\operatorname{Alg}(Z)$ contains a rationally strictly cyclic operator [15]. Since $\operatorname{Alg}(\mathscr{V})$ is also not inverse closed, we expect the same.

\section{STRONGLY STRICTLY CYCLIC OPERATOR ALGEBRAS}

Motivated by [15], we define an operator algebra $A$ to be strongly strictly cyclic if for every invariant subspace $M$ of $A, A$ restricted to $M$ is norm closed and strictly cyclic.

Theorem 2.1. Let $A$ be any strongly strictly cyclic operator algebra. Then $\operatorname{Lat}(A)$ satisfies the ascending chain condition (A.C.C.).

Proof. Suppose $0<P_{1}<P_{2}<\cdots$ is an ascending chain in $\operatorname{Lat}(A)$. Then $0<$ $P_{1}<P_{2}<\cdots<\bigvee_{n=1}^{\infty} P_{n}$ is a complete nest $\mathcal{N}$ in the subspace corresponding to the projection $\bigvee_{n=1}^{\infty} P_{n}$. Since $A$ restricted to the subspace is contained in $\operatorname{Alg}(\mathscr{N})$, we have that $\operatorname{Alg}(\mathcal{N})$ is strictly cyclic; so by Theorem $1.2, \mathscr{N}^{\perp}$ contains an atom. This is a contradiction.

The next main goal of this section is to characterize the strongly strictly cyclic reflexive operator algebras with a commutative subspace lattice and to give a "model" for the distributive lattices which are attainable by such algebras.

We begin with a discussion of some pertinent concepts which are well known. If $\mathscr{L}$ is a commutative subspace lattice and $P \in \mathscr{L}$, then we may define a commutative lattice acting in the subspace corresponding to $P^{\perp}$ by

$$
P^{\perp} \mathscr{L}=\left\{P^{\perp} Q=P^{\perp} \wedge Q: Q \in \mathscr{L}\right\} .
$$

To show that $P^{\perp} \mathscr{L}$ is strongly closed and hence a subspace lattice, assume that $P^{\perp} Q_{n} \rightarrow R$ where $Q_{n} \in \mathscr{L}$ for all $n$. Then

$$
\begin{aligned}
P \vee Q_{n} & =P \vee\left(\left(P \wedge Q_{n}\right) \vee\left(P^{\perp} \wedge Q_{n}\right)\right) \\
& =\left(P \vee\left(P \wedge Q_{n}\right) \vee\left(P^{\perp} \wedge Q_{n}\right)\right) \\
& =\left(P \vee\left(P^{\perp} \wedge Q_{n}\right)\right) \rightarrow P \vee R \in \mathscr{L} .
\end{aligned}
$$

Hence

$$
P^{\perp}(P \vee R)=P^{\perp} \wedge(P \vee R)=\left(P^{\perp} \wedge P\right) \vee\left(P^{\perp} \wedge R\right)=R
$$

We also define, for $P \in \mathscr{L}$, the commutative subspace lattice $P \mathscr{L}=\{P \wedge$ $Q: Q \in \mathscr{L}\}$.

Recall again that every reflexive operator algebra with a commutative subspace lattice is of the form $\operatorname{Alg}(\mathscr{L})$. 
Proposition 2.2. Let $\mathscr{L}$ be a commutative subspace lattice and let $M$ be the invariant subspace corresponding to some $P \in \mathscr{L}$. Then $\operatorname{Alg}(\mathscr{L})$ restricted to $M$ is $\operatorname{Alg}(P \mathscr{L})$. Hence $\operatorname{Alg}(\mathscr{L})$ restricted to $M$ is weakly closed.

Proof. Let $T \in \operatorname{Alg}(\mathscr{L})$. On the subspace of $H$ corresponding to $P$, we have $(P Q)^{\perp} T(Q P)=\left(P Q^{\perp}\right) T(Q P)=P Q^{\perp} T Q P=0$. Thus $\operatorname{Alg}(\mathscr{L})$ restricted to $M \subseteq \operatorname{Alg}(P \mathscr{L})$. Conversely, if $T \in \operatorname{Alg}(P \mathscr{L})$ then consider the operator $P T P$ defined on $H$. For any $Q \in \mathscr{L}$ we have $Q^{\perp}(P T P) Q=\left(Q^{\perp} P\right) T(P Q)=0$ on the spaces $P$ and $P^{\perp}$.

From the dual of Theorem 1.3 we know that $\operatorname{Alg}(\mathscr{L})$ is strictly cyclic iff there are a finite number $E_{1}, E_{2}, \ldots, E_{m}$ of maximal nonidentity projections such that for any $E \in \mathscr{L}, E \neq I, E \leq E_{i}$ for some $i$. It follows that $\operatorname{Alg}(\mathscr{L})$ is strongly strictly cyclic iff for each projection $P \in \mathscr{L}, P \neq 0$, there are maximal projections $E_{1}, E_{2}, \ldots, E_{m}<P$ such that for all $E<P, E \leq E_{i}$ for some $i$.

Hence $\operatorname{Alg}(\mathscr{L})$ is strongly strictly cyclic iff the subspace lattice $\mathscr{L}^{\perp}$ has the following property: For each $F \in \mathscr{L}^{\perp}, F \neq 0$, there exist $F_{1}, F_{2}, \ldots, F_{n} \in$ $\mathscr{L}^{\perp}$ that cover $F$, and for each $G \in \mathscr{L}^{\perp}$ with $F<G$ we have $F_{i} \leq G$ for some $i$.

Recall that for any lattice $\mathscr{L}$ and $a, b \in \mathscr{L}$, the interval $[a, b]=\{x \in$ $\mathscr{L}: a \leq x \leq b\}$.

Lemma 2.3. Let $\mathscr{L}$ be a commutative subspace lattice and $E \in \mathscr{L}$. The projection $E \in \mathscr{L}$ has a finite number of covers in $\mathscr{L}$ iff the lattice $E^{\perp} \mathscr{L}$ has a finite number of atoms.

Proof. The projection $E \in \mathscr{L}$ has a finite number of covers iff the interval lattice $[E, 1]$ has a finite number of atoms. Consider the map $\varphi: E^{\perp} \mathscr{L} \rightarrow$ $[E, 1]$ defined by $\varphi(A)=E \vee A$. This is an isomorphism with $\varphi^{-1}(B)=$ $E^{\perp} \wedge B$. The result follows.

Theorem 2.4. Let $\mathscr{L}$ be a commutative subspace lattice. Then $\operatorname{Alg}(\mathscr{L})$ is strongly strictly cyclic iff $\mathscr{L}$ satisfies the ascending chain condition (A.C.C.).

Proof. Assume that $\mathscr{L}^{\perp}$ satisfies D.C.C. We will show that for each $F \in \mathscr{L}^{\perp}$, $F \neq I$, there are covers $F_{1}, F_{2}, \ldots, F_{n}$ of $F$ such that for any $G>F, G \geq F_{i}$ for some $i$. From D.C.C. we know that any $G>F$ contains a cover of $F$. To show that there are only a finite number of covers of $F$ we use Lemma 2.2 and show that the subspace lattice $F^{\perp} \mathscr{L}^{\perp}$ has only a finite number of atoms. The map $\Psi: \mathscr{L}^{\perp} \rightarrow F^{\perp} \mathscr{L}^{\perp}$ defined by $\Psi(Q)=F^{\perp} Q$ is a lattice epimorphism. Therefore $F^{\perp} \mathscr{L}^{\perp}$ satisfies D.C.C. If $F^{\perp} \mathscr{L}^{\perp}$ contained an infinite number of atoms, then it would contain an infinite complete atomic Boolean lattice of projections on a separable Hilbert space. Such a Boolean lattice is isomorphic to the lattice of subsets of the natural numbers which does not have the descending chain condition. The converse follows from Theorem 2.1. 
The theorem does not hold for general reflexive algebras as the following example shows. Let $H=H_{1} \oplus H_{2}$ where $\operatorname{dim} H_{1}<\infty$, and let $A$ be the algebra of all matrices of the form

$$
\begin{aligned}
& H_{1} \quad H_{2} \\
& {\left[\begin{array}{ll}
\lambda & A \\
0 & B
\end{array}\right]}
\end{aligned}
$$

where $\lambda \in \mathbf{C}$ and $A, B$ are arbitrary. Then $A$ is reflexive and $\operatorname{Lat}(A)$ satisfies A.C.C. but $A$ is not strongly strictly cyclic.

Let $\mathscr{L}$ be a complete lattice. By a representation of $\mathscr{L}$ we mean a homomorphism $\pi: \mathscr{L} \rightarrow P(H)$, the projections on $H$ such that

$$
\pi(0)=0, \quad \pi(1)=1, \quad \pi\left(\bigvee a_{i}\right)=\bigvee \pi\left(a_{i}\right), \quad \pi\left(\bigwedge a_{i}\right)=\bigwedge \pi\left(a_{i}\right) .
$$

A complete lattice $\mathscr{L}$ is said to be attainable if there is a faithful representation $\pi$ and an operator algebra $A$ such that $\pi(\mathscr{L})=\operatorname{Lat}(A)$.

A problem of longstanding interest in operator theory has been the characterization of those lattices which are attainable [14]. Although in its full generality this problem is extremely difficult, if not impossible, considerable progress can be made in certain cases. Thus Arveson [1] characterized the distributive lattices which are attainable by reflexive operator algebras on a separable Hilbert space as those which are countably generated as a complete lattice and admit a faithful normal valuation [1]. We will obtain a more concrete description of the distributive lattices which are attainable by strongly strictly cyclic reflexive algebras. Throughout the remainder of this section, $\mathscr{L}$ will denote a commutative subspace lattice.

By an interval of $\mathscr{L}$ we mean a projection of the form $P-Q$ where $P, Q \in$ $\mathscr{L}$ and $P \geq Q$. If $P-Q$ and $A-B$ are intervals, then the projection $(P-Q)(A-B)=P A+Q B-Q A-P B=P A-(Q A+P B-Q B)=P A-(Q A \vee P B)$, which is an interval. A nonzero interval is called an atomic interval if it does not properly contain another nonzero interval. Note that if $A-B$ is an atomic interval and $P-Q$ is any interval, then $(A-B)(P-Q)=0$ or $(A-B)(P-Q)=$ $A-B$.

Lemma 2.5. Let $\mathscr{C}$ be a maximal chain in $\mathscr{L}$ and let $P-Q$ be an atomic interval in $\mathscr{C}$. Then $P-Q$ is an atomic interval in $\mathscr{L}$.

Proof. If $L \in \mathscr{L}$, let $M=(L \vee Q) \wedge P$. Then $Q \leq M \leq P$ so $M \in \mathscr{C}$ and thus $M=P$ or $M=Q$. Hence $L(P-Q)=M(P-Q)$ is 0 or $P-Q$. It follows that for any interval $A-B$ of $\mathscr{L},(A-B)(P-Q)=0$ or $P-Q$. Thus $P-Q$ is an atomic interval of $\mathscr{L}$.

Lemma 2.6. Let $\mathcal{N}$ be a complete nest (with 1) satisfying D.C.C. and let $\left\{E_{i}\right\}$ be the collection of atomic intervals of $\mathscr{N}$. Then $\bigvee E_{i}=1$.

Proof. Let $P=\bigvee E_{i}$. We will show that for all $M \in \mathscr{N}, M \leq P$. In particular, $1 \leq P$. If there is an $M \in \mathcal{N}$ with $N \Varangle P$ then there is a smallest such $M$. 
Let $Q=\bigvee_{R<M} R$. Clearly $Q \leq P$ and $M-Q$ is an atomic interval of $\mathscr{N}$. We have $(M-Q) \vee Q=M$. But $M-Q \leq P$ and $Q \leq P$, so $M \leq P$, which is a contradiction. Thus $P=1$.

Now let $\operatorname{Alg}(\mathscr{L})$ be strongly strictly cyclic. Then $\mathscr{L}^{\perp}$ satisfies D.C.C. Take $\mathscr{C}$ to be any maximal nest in $\mathscr{L}^{\perp}$. Then there are atomic intervals $E_{1}, E_{2}, \ldots$ of $\mathscr{C}$ such that $\bigvee E_{n}=1$. Furthermore $E_{1}, E_{2}, \ldots$ are atomic intervals of $\mathscr{L}^{\perp}$ by Lemma 2.5. Hence for any $P \in \mathscr{L}^{\perp}$ and any $n$, either $P E_{n}=E_{n}$ or $P E_{n}=0$ since $P$ itself is an interval. It follows from $\bigvee E_{n}=1$ that $P=$ $\bigvee_{E_{n} \leq P} E_{n}$, the representation of $P$ as the join of orthogonal atomic intervals, is unique. It follows that $\mathscr{L}^{\perp}$ is a complete sublattice of the complete atomic Boolean lattice generated by the $E_{n}$ 's.

Consider the lattice $\mathscr{P}(N)$ of subsets of the natural numbers. We view $\mathscr{P}(N)$ as characteristic functions with the complete metric

$$
d\left(\chi_{A}, \chi_{B}\right)=\sum_{n=1}^{\infty} \frac{\left|\chi_{A}(n)-\chi_{B}(n)\right|}{2^{n}} .
$$

This metric gives the topology of pointwise convergence on $\mathscr{P}(N)$ and turns $\mathscr{P}(N)$ into a topological lattice which may be identified with the topological lattice $\{0,1\} \times\{0,1\} \times \cdots$ and the product topology.

Let $\mathscr{B}$ be any infinite complete atomic Boolean lattice of projections on a separable Hilbert space. And give $\mathscr{B}$ the strong operator topology. Then $\mathscr{P}(N)$ is lattice isomorphic to $\mathscr{B}$ in a natural manner. Moreover, the strong operator topology on $\mathscr{B}$ coincides with the metric topology defined above for $\mathscr{P}(N)$. Hence we observe that $\mathscr{B}$ is completely distributive and also compact in the strong operator topology. We have proved the following theorem.

Theorem 2.7. An abstract distributive lattice $\mathscr{L}$ is attainable by a strongly strictly cyclic reflexive algebra iff it is isomorphic to a complete sublattice of $\{0,1\} \times$ $\{0,1\} \times \cdots$ that satisfies the A.C.C.

Corollary 2.8. If $\operatorname{Alg}(\mathscr{L})$ is strongly strictly cyclic, then (i) $\mathscr{L}$ is completely distributive, and (ii) $\mathscr{L}$ is compact in the strong operator topology.

We remark that a sublattice of $\{0,1\} \times\{0,1\} \times \cdots$ is complete iff it is closed in the product topology. Also (ii) is a consequence of (i) for every commutative subspace lattice.

Recall that the adjoint algebra $A^{*}$ of an operator algebra $A$ is $\left\{T^{*}: T \in A\right\}$.

Proposition 2.9. Both $\operatorname{Alg}(\mathscr{L})$ and $\operatorname{Alg}\left(\mathscr{L}^{\perp}\right)=\operatorname{Alg}(\mathscr{L})^{*}$ are strongly strictly cyclic iff $\mathscr{L}$ is a finite lattice.

Proof. Take a maximal chain $\mathscr{C}$ in $\mathscr{L}$. Then $\mathscr{C}$ satisfies both A.C.C. and D.C.C. so $\mathscr{C}$ is finite. Hence $\mathscr{C}$ has only a finite number of atomic intervals and so $\mathscr{L}$ is finite. The converse is immediate. 


\section{STRICTLY CYCLIC OPERATORS}

Consider the algebra of infinite lower triangular matrices. It follows from [15] that this algebra contains strictly cyclic operators. It is natural to consider the more general question of when a strictly cyclic operator algebra contains a strictly cyclic operator. For operator algebras of the form $\operatorname{Alg}(\mathscr{L})$, this has the following importance. Recall that if $T$ is strictly cyclic then there is a lattice isomorphism between the invariant subspaces of $T$ and the closed ideals of $\mathscr{U}_{T}$, the norm-closed algebra generated by $T$. This correspondence takes the form

$$
M \leftrightarrow I_{M} x,
$$

where $x$ is a strictly cyclic vector for $T$ and $I_{M}=\left\{S \in \mathscr{U}_{T}: S x \in M\right\}$. Hence, if $T \in \operatorname{Alg}(\mathscr{L})$ is strictly cyclic then there is a lattice monomorphism $\varphi: \mathscr{L} \rightarrow \mathscr{I}\left(\mathscr{U}_{T}\right)$, the lattice of closed ideals of $\mathscr{U}_{T}$. Moreover $\varphi$ preserves arbitrary meets and joins.

First we give an example of a commutative subspace lattice $\mathscr{L}$ for which $\operatorname{Alg}(\mathscr{L})$ is strictly cyclic but contains no strictly cyclic operators.

Definition 3.1. An operator $T$ is called co-strictly cyclic if $T^{*}$ is strictly cyclic.

The next result is a particular case of [8, Lemma 10].

Iemma 3.2. If $T$ is strictly cyclic then the restriction of $T^{*}$ to any $M \in \operatorname{Lat}\left(T^{*}\right)$ is co-strictly cyclic.

Proof. We have

$$
\begin{aligned}
& \begin{array}{lll}
M & M^{\perp} \quad M \quad M^{\perp}
\end{array} \\
& T^{*}=\left[\begin{array}{cc}
A^{*} & B^{*} \\
0 & C^{*}
\end{array}\right] \text { and } T=\left[\begin{array}{cc}
A & 0 \\
B & C
\end{array}\right] .
\end{aligned}
$$

Let $\left[\begin{array}{l}x_{1} \\ x_{2}\end{array}\right]$ be a strictly cyclic vector for $T$. Given $y \in M$, there is a sequence $\left\{P_{n}\right\}$ of polynomials such that

$$
P_{n}(T)\left[\begin{array}{l}
x_{1} \\
x_{2}
\end{array}\right] \rightarrow\left[\begin{array}{l}
y \\
0
\end{array}\right]
$$

and $P_{n}(T)$ converges in norm to some element of $\mathscr{U}_{T}$. Therefore $P_{n}(A) x_{1} \rightarrow y$ and $P_{n}(A)$ converges in norm to some element of $\mathscr{U}_{A}$. Hence $A$ is strictly cyclic.

Lemma 3.3. Let $\operatorname{dim} H=\infty$. There are no strictly cyclic operators of the form

$$
T=\left[\begin{array}{cc}
\mathbf{C} & H \\
0 & 0 \\
B & \lambda I
\end{array}\right]
$$

where $\lambda \in \mathbf{C}$.

Proof. Calculation yields $T(\lambda-T)=0$ and there are no algebraic strictly cyclic operators when $\operatorname{dim} H=\infty$. 
For the sake of clarity and completeness we sketch a proof of the well-known result that if $\lambda \in \sigma(T)$ then $\bar{\lambda} \in \sigma_{p}\left(T^{*}\right)$ for strictly cyclic $T$ [13, Lemma 3.4]. Suppose that $\lambda \in \sigma(T)$. Then there is a multiplicative linear functional $\varphi: \mathscr{U}_{T} \rightarrow \mathbf{C}$ such that $\varphi(T)=\lambda$. Let $p: \mathscr{U}_{T} \rightarrow H$ be defined by $p(B)=B x$, where $x$ is a strictly cyclic vector for $T$. Then we obtain a linear functional $l$ on $H$

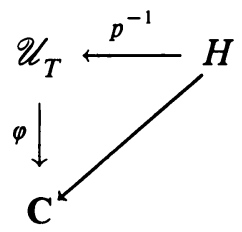

Hence for some vector $y \in H, l(x)=(x, y)$. Therefore $\varphi(B)=(B x, y)$ for $B \in \mathscr{U}_{T}$. Now $(T B x, y)=\varphi(T B)=\varphi(T) \varphi(B)=\lambda(B x, y)$, so $\left(B x, T^{*} y\right)=$ $(B x, \bar{\lambda} y)$ and therefore $\left(B x, T^{*} y-\bar{\lambda} y\right)=0$ for every $B \in \mathscr{U}_{T}$. This implies that $T^{*} y=\bar{\lambda} y$.

For the example, let $H=\mathrm{C} \oplus L^{2}[0,1]$. For each Borel set $B \subseteq[0,1], P_{B}$ is the projection $f \rightarrow \chi_{B} f$. The commutative subspace lattice $\mathscr{L}$ consists of all projections of the form $0 \oplus P_{B}$ together with the identity projection. With respect to the above decomposition of $H, \operatorname{Alg}(\mathscr{L})$ consists of all operators of the form

$$
\begin{aligned}
& \text { C } \\
& L^{2} \\
& {\left[\begin{array}{cc}
a & 0 \\
B & M_{f}
\end{array}\right] \quad \text { where } a \in \mathbf{C}}
\end{aligned}
$$

and where $f \in L^{\infty}[0,1]$ gives $M_{f}$, the operator of multiplication by $f$.

Then $\operatorname{Alg}(\mathscr{L})$ is strictly cyclic with strictly cyclic vector $1 \oplus 0$. We will show that $\operatorname{Alg}(\mathscr{L})$ does not contain a strictly cyclic operator. By translation, assume that $T=\left[\begin{array}{cc}0 & 0 \\ B & M_{f}\end{array}\right]$ is strictly cyclic. If $\lambda \neq 0 \in \sigma\left(M_{f}\right)$ then there is a sequence $h_{n} \in L^{2}[0,1]$ with $\left\|h_{n}\right\|_{2}=1$ and $\left\|M_{f} h_{n}\right\|_{2} \rightarrow 0$. Hence $\left\|T\left(0 \oplus h_{n}\right)\right\|_{2} \rightarrow 0$, so $\lambda \in \sigma(T)$. Therefore $\bar{\lambda}$ is an eigenvalue of $T^{*}=\left[\begin{array}{ll}0 & B^{*} \\ 0 & M_{\bar{f}}\end{array}\right]$. So for some $z \oplus g$ we have $T^{*}(z \oplus g)=\bar{\lambda}(z \oplus g)$. Since $\lambda \neq 0, g \neq 0$ and it follows that $M_{\bar{f}} g=\bar{\lambda} g$. Therefore $\bar{f}=\bar{\lambda}$ on some set $B$ of positive measure. Now restrict $T^{*}$ to the subspace $1 \oplus P_{B} \in \mathscr{L}^{\perp}$. This restriction has the form $\left[\begin{array}{l}0 \\ 0 \\ 0\end{array}\right]$, which is co-strictly cyclic, so $\left[\begin{array}{cc}0 & 0 \\ R^{*} & \lambda I\end{array}\right]$ must be strictly cyclic. This contradicts Lemma 3.3. Hence $\operatorname{Alg}(\mathscr{L})$ contains no strictly cyclic operators.

A strictly cyclic operator algebra, which in some respects is similar to the previous example, consists of all matrices of the form

$$
\left[\begin{array}{cccc}
a_{1} & & & \\
x_{2} & a_{2} & & 0 \\
x_{3} & & . & \\
\vdots & 0 & & .
\end{array}\right]
$$


which define bounded operators. It follows from [8, p. 12] that this algebra contains a strictly cyclic operator. This leads us to suppose that whether or not $\operatorname{Alg}(\mathscr{L})$ contains a strictly cyclic operator is related to the "discreteness" of $\mathscr{L}$. However the situation seems quite complicated and we have only been able to prove the forthcoming Theorem 3.6.

Lemma 3.4. Let $T_{1}, T_{2}$ be strictly cyclic operators with $\sigma\left(T_{1}\right) \cap \sigma\left(T_{2}\right)=\varnothing$. Then $T_{1} \oplus T_{2}$ is strictly cyclic.

Proof. According to [7, Theorem 4(iii)], $\sigma\left(T_{i}\right), i=1,2$, has no "holes". The result follows from the Riesz-Dunford functional calculus.

Recall that if $\mathscr{M}$ is an atomic m.a.s.a. then there is an orthonormal basis, $e_{1}, e_{2}, \ldots$ of $H$, such that $\mathscr{M}$ is weakly spanned by the projections $e_{1} \otimes e_{1}$, $e_{2} \otimes e_{2}, \ldots$.

Lemma 3.5. Let $\mathscr{M}$ be an atomic m.a.s.a. and let $P_{1}, P_{2}, \ldots, P_{n}$ be projections in $\mathscr{M}$ such that for each $i, e_{i} \otimes e_{i} \leq P_{i}$. Then there are pairwise orthogonal projections $Q_{1}, \ldots, Q_{n} \in \mathscr{M}$ such that $e_{i} \otimes e_{i} \leq Q_{i} \leq P_{i}$ for all $i$ and $\bigvee_{i=1}^{n} Q_{i}=$ $\bigvee_{i=1}^{n} P_{i}$.

Proof. The lattice of $\mathscr{M}$ is isomorphic to the power set of the natural numbers where the set-theoretic equivalent is obvious.

Theorem 3.6. Suppose that $\operatorname{Alg}(\mathscr{L})$ is strictly cyclic and that $\mathscr{L} \subseteq$ atomic m.a.s.a. Then $\operatorname{Alg}(\mathscr{L})$ contains a strictly cyclic operator.

Proof. Suppose $\operatorname{Alg}(\mathscr{L})$ is strictly cyclic and let $F_{1}, \ldots, F_{n}$ be the atoms of $\mathscr{L}^{\perp}$. Since $\mathscr{L}^{\perp} \subseteq \mathscr{M}$, each atom of $\mathscr{L}^{\perp}$ is spanned by some of the vectors $e_{1}, e_{2}, \ldots$ Suppose that $e_{1}, e_{2}, \ldots$ are labelled so that $F_{i} e_{i}=e_{i}$ for $i=$ $1,2, \ldots, n$. As in the proof of Theorem 1.3, for any $x_{i} \in A \bar{e}_{i}$ we know that $x_{i} \otimes e_{i} \in \operatorname{Alg}(\mathscr{L})$. Let $P_{i}$ be the projection in $\mathscr{L}$ corresponding to the subspace $A \bar{e}_{i}$. Note that $e_{i} \otimes e_{i} \leq P_{i}$. By Lemma 3.5 there are pairwise orthogonal projections $Q_{1}, \ldots, Q_{n} \in \mathscr{M}$ such that $Q_{1} \vee \cdots \vee Q_{n}=1$ and $e_{i} \otimes e_{i} \leq Q_{i} \leq P_{i}$ for all $i$. Therefore, if $Q_{i} y_{i}=y_{i}$ then $y_{i} \otimes e_{i} \in \operatorname{Alg}(\mathscr{L})$.

There is a partition $S_{1} \cup \cdots \cup S_{n}$ of the natural numbers such that $Q_{i}=$ $\sum_{k \in S_{i}} e_{k} \otimes e_{k}$ and $i \in S_{i}$. Consider an operator of the form

$$
T_{i}=y_{i} \otimes e_{i}+\sum_{k \in S_{i}} c_{k} e_{k} \otimes e_{k}
$$

where $Q_{i} y_{i}=y_{i}$ and the $c_{k}$ 's are complex numbers. Since

$$
\sum_{k \in S_{i}} c_{k} e_{k} \otimes e_{k} \in \mathscr{M} \subseteq \operatorname{Alg}(\mathscr{L}),
$$

$T_{i} \in \operatorname{Alg}(\mathscr{L})$. Therefore $T_{1}+\cdots+T_{n} \in \operatorname{Alg}(\mathscr{L})$. 
It is easy to verify that $T_{1}+\cdots+T_{n}$ commutes with each $Q_{i}$, indeed $T_{i}$ vanishes on $Q_{i}^{\perp}$, so that the sum is actually $T_{1} \oplus T_{2} \oplus \cdots \oplus T_{n}$. We need to choose the spectra of each $T_{i}$ correctly (Lemma 3.4) and each $T_{i}$ to be strictly cyclic. But each $T_{i}$ has the form

$$
\left[\begin{array}{cccc}
a_{1} & & & \\
x_{2} & a_{2} & & 0 \\
x_{3} & & \cdot \\
\cdot & 0 & & \cdot
\end{array}\right]
$$

on the subspace corresponding to $Q_{i}$ so that $T_{1}, T_{2}, \ldots, T_{n}$ can be chosen to yield a strictly cyclic operator on $\bigvee_{i=1}^{n} Q_{i}=H[8, \mathrm{p} .12]$.

Corollary 3.7. If $\operatorname{Alg}(\mathscr{L})$ is strongly strictly cyclic, then $\operatorname{Alg}(\mathscr{L})$ contains a strictly cyclic operator.

Proof. We have seen that $\mathscr{L} \subset$ atomic Boolean lattice $\subseteq$ atomic commutative $W^{*}$ algebra. The result follows from the fact that every atomic commutative $W^{*}$-algebra can be extended to an atomic m.a.s.a.

It would be desirable to obtain a characterization of the commutative subspace lattices which are attainable by strictly cyclic operators.

Acknowledgments. We are deeply indebted to D. A. Herrero for reading the manuscript and offering several important corrections, as well as to the referee for pointing out a number of obscurities and simpler proofs. The referee has also informed us that the last several lines of the proof of Theorem 1.3 are due to Longstaff in a different context.

\section{REFERENCES}

1. W. Arveson, Operator algebras and invariant subspaces, Ann. of Math. 100 (1974), 433-532.

2. B. Barnes, Operators with a strictly cyclic vector, Proc. Amer. Math. Soc. 41 (1973), 480-486.

3. K. R. Davidson, Commutative subspace lattices, Indiana Univ. Math. J. 27 (1978), 479-490.

4. __ Nest algebras, Pitman Res. Notes Mat., Longman, 1988.

5. J. Froelich, Compact operators, invariant subspaces and spectral synthesis, J. Funct. Anal. 81 (1988), 1-37.

6. G. Gratzer, Lattice theory, Freeman, San Francisco, Calif., 1971.

7. D. A. Herrero, Operator algebras of finite strict multiplicity, Indiana Univ. Math. J. 22 (1972), 13-24.

8. $\frac{}{9-18}$, Operator algebras of finite strict multiplicity. II, Indiana Univ. Math. J. 27 (1978),

- Triangular strictly cyclic operators, Integral Equations Operator Theory 10 (1987), 297-303.

10. D. A. Herrero and A. L. Lambert, On strictly cyclic algebras, P-algebras, and reflexive operators, Trans. Amer. Math. Soc. 185 (1973), 229-235.

11. A. Hopenwasser, The equation $T x=y$ in reflexive operator algebras, Indiana Univ. Math. J. 29 (1980), 121-126. 
12. __ Hilbert-Schmidt interpolation in CSL-algebras, Preprint.

13. A. Lambert, Strictly cyclic operator algebras, Pacific J. Math. 39 (1971), 717-726.

14. H. Radjavi and P. Rosenthal, Invariant subspaces, Ergeb. Math., vol. 77, Springer-Verlag, New York, Heidelberg, and Berlin, 1973.

15. A. Shields, Weighted shift operators and analytic function theory, Topics in Operator Theory, Math. Surveys, no. 13, Amer. Math. Soc., Providence, R.I., 1974.

Department of Mathematics, University of Houston, University Park, Houston, TEXAS 77204 